\title{
Sedimentation of lithium-iron-phosphate and carbon black particles in opaque suspensions used for lithium- ion-battery electrodes
}

\author{
R. Balbierer ${ }^{1, *}$, R. Gordon ${ }^{1}$, S. Schuhmann ${ }^{1}$, N. Willenbacher ${ }^{1}$, H. Nirschl ${ }^{1}$, and \\ G. Guthausen ${ }^{1,2}$ \\ ${ }^{1}$ Institute of Mechanical Process Engineering and Mechanics, Karlsruhe Institute of Technology (KIT), Straße am Forum 8, \\ 76131 Karlsruhe, Germany \\ ${ }^{2}$ Chair of Water Chemistry and Water Technology, Karlsruhe Institute of Technology (KIT), Engler-Bunte-Ring 9a, 76131 Karlsruhe, \\ Germany
}

\begin{abstract}
Sedimentation of opaque suspensions of carbon black and lithium-iron-phosphate was investigated by spin-echo-based magnetic resonance imaging. Optical methods are usually applied to determine settling velocities, but are limited with respect to high concentrations and optical transparency. The presented method uses intensity data from the noninvasively measured magnetic resonance signal of the sample. The settling velocity is obtained from the evolution of the signal intensity profiles based on the contrast in ${ }^{1} \mathrm{H}$ magnetic resonance imaging between particles and liquid. New insights into the sedimentation in opaque suspensions are provided, since the ${ }^{1} \mathrm{H}$ images uncover the spatial distribution of the particles and its agglomerates, as well as the shape of the settling front. Additionally, the sedimentation was experimentally studied using a sedimentation balance, which gravimetrically measures the increase in mass fraction over time due to the settling of particles. By parallel usage of these two methods, the sedimentation processes of opaque suspensions of lithium-ionbattery electrode materials were investigated. The sedimentation balance covers high, technically relevant concentrations. Limiting factors of the methods are discussed, which are mainly signal intensity in the magnetic resonance imaging and the increasing viscosity of highly concentrated suspensions.
\end{abstract}




\section{Introduction}

Gravity-driven sedimentation processes of carbon black (CB) and lithium-iron-phosphate (LFP) particles were observed by magnetic resonance imaging (MRI) and a sedimentation balance. These particles are increasingly applied as active material in lithiumion-battery (LIB) electrodes.

The majority of LIB utilizes cobalt-based cathode materials as well as aluminum, manganese and nickel blends to fulfill the dominant application demands [1]. New approaches insert silicon, tin or germanium into the active materials of the anode, which reduces the mechanical stress during lithiation, but enlarges the effective expansion volume [2-5]. However, it remains to future generations of LIBs whether the cyclical and calendrical stability of these materials meets the application requirements [6-9]. With regard to safety, LFP-based cathodes deliver undisputable advantages, in cases where capacity is the less dominant requirement. The lack of required conductivity and energy density/capacity of LFP is addressed by coating the nanoscale LFP particles with CB as well as adding CB nanoparticles [10-12]. Thus, LFP currently is a commonly used electrode material with respect to well-balanced capacity, as well as good safety properties and economic factors.

The slurries of the active material are steadily under improvement, due to the rising demand of increasing capacity and cyclic durability. To increase the homogeneity of LFP and CB particle distribution in the slurry, the particle systems of electrode materials are of general interest [13, 14]. Gravity-driven sedimentation occurs and affects the homogeneity of particles distribution.

Over decades, the sedimentation of particles that vary in shape and kinematic properties was investigated. Several theoretical models were published and described the experimental data within given limits [15-20]. The sedimentation itself can be understood as the movement of particles in fluids due to external forces, e.g., due to gravitational or centrifugal forces. Important for the motion in a suspension are certainly the density difference between particles and liquid, the particle volume fraction and their shape, and the viscosity. Several techniques were developed to optically study sedimentation [21-23]. The limitations of the optical methods are obvious: a certain amount of light needs to reach a photosensitive detector to measure the amount and rate of particle movement. Concentration is limited as hindered settling occurs due to particle interactions [24]. Suspensions with LFP and CB tend to become opaque even for low particle mass fractions $\left(\phi_{\mathrm{p}}<0.5 \%\right)$. Hence, optical measurements are limited regarding this class of materials.

MRI was used to explore sedimentation of $C B$ and LFP in these opaque suspensions. As the particles themselves do not provide a ${ }^{1} \mathrm{H}-\mathrm{MRI}$ signal, the MR intensity is due to the liquid in the suspension, which was silicon oil. The versatility of the MRI approach is emphasized as only a single suitable and nuclear magnetic resonance (NMR) active fluid needs to be applied to measure both particle classes in situ. Signal intensities were measured spatially and time-resolved to observe the sedimentation at the bottom of an NMR tube, caused by settling particles with different particle size and agglomeration, i.e., the settling front (SF). In addition, a sedimentation balance was built to characterize the evolution of mass fraction over time. By parallel usage of these two methods, velocities for CB and LFP at a given viscosity can be measured and the final slurry composition with the required particle fractions can be characterized in terms of sedimentation.

\section{Materials}

In order to achieve one suitable viscosity for both particle classes, a silicon oil (polydimethylsiloxane (PDMS), Quax GmbH, Germany) was used as fluidic phase in the suspensions for the ${ }^{1} \mathrm{H}$-MRI measurements. This choice allows the sedimentation to take place but simultaneously enlarges the timescale for the measurements. Carbon-coated LFP, i.e., $\mathrm{LiFePO}_{4}$ particles and CB particles were used as received. Due to the experimental state of the slurry recipe, the materials supplier is not mentioned. The values for the specific surface area (SSA) for LFP were provided. According to the manufacturer, the mean size of the primary CB particles is approximately $42 \mathrm{~nm}$. However, CB and LFP particles tend to agglomerate, reaching an average size of a few microns. Dispersed $\mathrm{CB}$ and LFP particles in PDMS also show this trend to agglomeration and increase the viscosity as a function of volume fraction $[25,26]$. The density of the solid materials was determined using a helium pycnometer (Micromeritics, Multi Volume Pycnometer, Karlsruhe, Germany). 
The targeted particle mass fraction for the MRI experiments was $\phi_{\mathrm{p}}=0.5 \mathrm{wt} \%$. This was achieved by a PDMS mass of $m_{\mathrm{f}}=859.4 \mathrm{mg} \pm 1.3 \%$ and a particle mass of $m_{\mathrm{p}}=4.25 \mathrm{mg} \pm 5.8 \%$. This results in an overall sample particle mass fraction of $\phi_{\mathrm{p}}=0.495 \pm 0.036 \mathrm{wt} \%$, which deviates from the targeted particle mass fraction by $\pm 7.2 \%$. Four samples of each material class were investigated by MRI (Table 1).

The equivalent sphere-particle size distribution (PSD) was determined by Fraunhofer diffraction (HELOS H0309, Sympatec GmbH, Clausthal-Zellerfeld, Germany) using an ultrasonic wet dispersing unit (QUIXEL, Sympatec GmbH, Clausthal, Germany) for dispersing the LFP and CB particles in acetone and ethanol, respectively (Fig. 1).

The same PDMS was used for the experiments with the sedimentation balance. In contrast to the MRI measurements, the particle concentrations were vastly increased. Experiments with particle mass fractions of $\phi_{\mathrm{m}}=10,30$ and $50 \mathrm{wt} \%$ for LFP were performed. The corresponding LFP suspensions had a low shear $\left(\dot{\gamma}=2.2 \mathrm{~s}^{-1}\right)$ viscosity of $\eta=0.1,1.5$ and 12.9 Pas, respectively (Fig. 2).

Investigations of higher particle mass fractions with $C B$ failed due to the increase in viscosity and the thus reduced and presumable absent sedimentation. Particle mass fractions of $\phi_{\mathrm{m}}>1 \%$ for CB already showed the mentioned behavior. As CB is mainly added to increase the conductivity of LIB electrodes, low mass fractions of CB are realized in real applications. Hence, only three different mass fractions of LFP were investigated by the sedimentation balance,

Table 1 Material properties of used fluid and particles

\begin{tabular}{lll}
\hline Fluid & & Polydimethylsiloxane (PDMS) \\
\hline$\rho\left(\vartheta=25^{\circ} \mathrm{C}\right)$ & & $0.955 \mathrm{~g} \mathrm{~cm}^{-3}$ \\
$v$ & & $20 \mathrm{~mm}^{2} \mathrm{~s}^{-1}$ \\
$\eta$ & & $19.1 \mathrm{mNsm}^{-2}$ \\
\hline Particles & Carbon black (CB) & Lithium-iron-phosphate \\
& & $(\mathrm{LFP})$ \\
\hline$x_{50,3}$ & $5.47 \mu \mathrm{m}$ & $1.71 \mu \mathrm{m}$ \\
rel.span & 2.96 & 5.24 \\
$\rho$ & $1.73 \mathrm{~g} \mathrm{~cm}{ }^{-3}$ & $3.51 \mathrm{~g} \mathrm{~cm}^{-3}$ \\
SSA & - & $11.6 \mathrm{~m}^{2} \mathrm{~g}^{-1}$ \\
Sample & CB_1, CB_2, CB_3, & LFP_1, LFP_2, LFP_3, \\
\multicolumn{1}{l}{ notation } & CB_4 & LFP_4 \\
\hline
\end{tabular}

due to LFP being gravimetrically the main ingredient for the active material of an LFP-based LIB. Additionally, a practically relevant composition of an electrode slurry, used for LIB, was investigated by the sedimentation balance with respect to its sedimentation behavior. This composition is based on the experimental recipe of a LFP-based aqueous active material slurry containing carboxymethylcellulose $(\mathrm{CMC})$ with a molecular weight $M_{\mathrm{W}}=700 \mathrm{kDa}$ and a degree of substitution of DS $=0.9$ as polymeric binder. The slurry had a low shear $\left(\dot{\gamma}=2.2 \mathrm{~s}^{-1}\right)$ viscosity of $\eta=14.3$ Pas.

\section{Experimental and methods}

With the help of an in-house-manufactured cylindrical polytetrafluoroethylene (PTFE) sample holder, two NMR tubes were placed side by side into a radiofrequency probe of the NMR spectrometer, i.e., into the field of view (FOV) (Fig. 3). A sagittal slice across the center of the tubes was measured by MRI. A time series of experiments with identical parameters and orientation allows the measurement of the sedimentation. Due to the NMR properties of the particles, i.e., especially their fast transverse relaxation, the images are negative images, i.e., the measured intensity is due to the liquid.

The materials were separately inserted into an NMR tube after it was filled with $900 \mu \mathrm{l}$ of PDMS. After coarsely dispersing the particles at the top, the tubes were placed into the probe of a Bruker $200 \mathrm{MHz}$ Avance III HD spectrometer. The maximum gradient for imaging was $1.5 \mathrm{Tm}^{-1}$. The MICWB40 probe with a ${ }^{1} \mathrm{H}$ birdcage allowed for a maximum outer sample diameter of $20 \mathrm{~mm}$. Hence, multiple NMR tubes with outer diameter of $5 \mathrm{~mm}$ could be measured simultaneously (Fig. 3).

\section{Spin-echo-based MRI}

The spin-echo-based pulse sequence RARE (rapid acquisition with relaxation enhancement) was used for the MRI experiments with parameters specific for the materials and application (Table 2). The advantage of RARE, with respect to the often faster gradient-echo based methods, is the reduced sensitivity toward magnetic susceptibility differences between materials, especially along the phase direction in the images. The samples were measured applying a 2D 

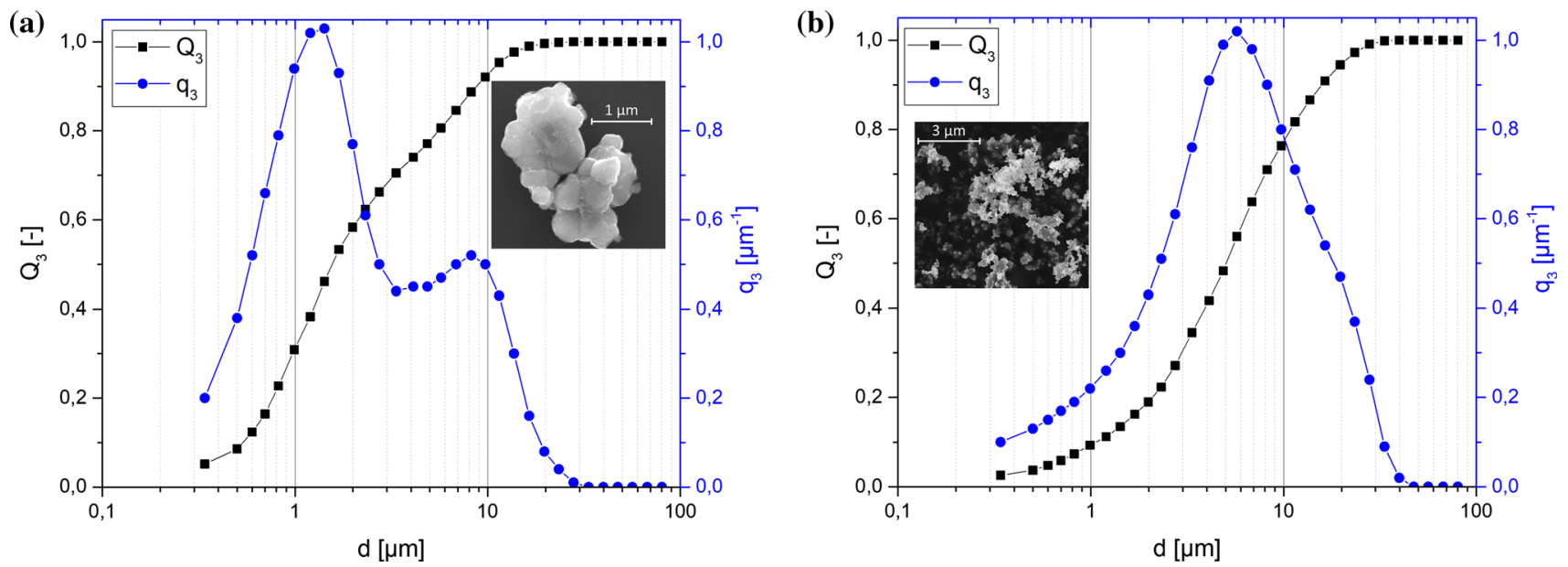

Figure 1 Cumulated particle size distribution $\left(Q_{3}\right)$ and its density $\left(q_{3}\right)$ of LFP (a) and CB (b). The insets show SEM images of a particle agglomerate of the respective material.

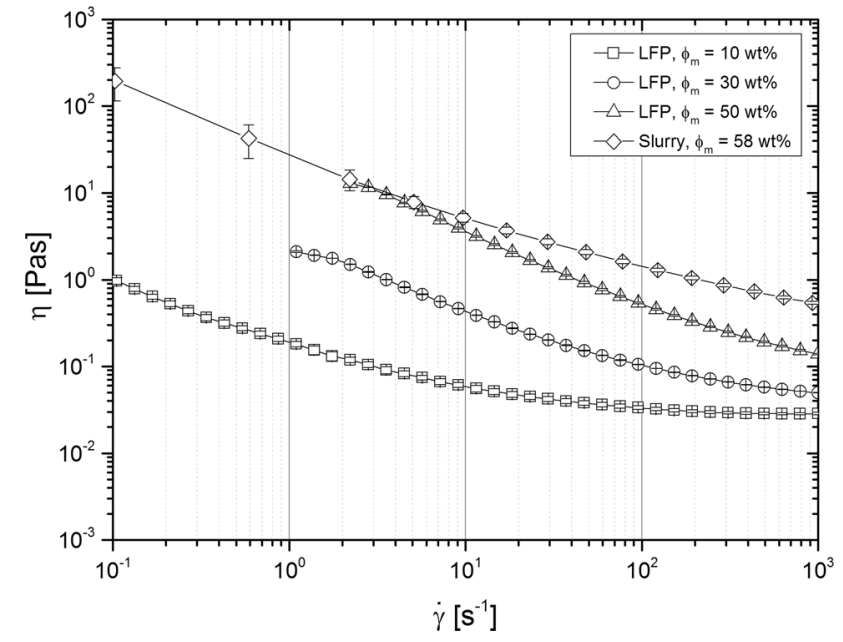

Figure 2 The viscosity $\eta$ depends on the shear rate $\dot{\gamma}$ of the slurry and the LFP suspensions with different mass fractions $\phi_{\mathrm{m}}$. The lines are to guide the eye.

RARE sequence with slice selection. Thus, the MRI signal intensity evolution is a measure to the SF in the opaque suspensions. The acquisition time given by the timestamp of the MRI raw data was used as time axis of sedimentation.

The aim of the study was to deduce the sedimentation velocity from the MR images. Mean values of the signal intensity were calculated. Only the image area in the middle of the tubes was considered. Boundary or wall effects near the tube's wall were therefore eliminated.

The wall effects are neglected with the argument that the ratio between the average particle diameter $x$ obtained from PSD and the inner tube diameter of approximately $d=4.09 \mathrm{~mm}$ is relatively small [27]. The ratios for LFP and CB are: $\{x / d\}_{\mathrm{LFP}}=4.16 \times 10^{-4}$ and $\{x / d\}_{\mathrm{CB}}=1.34 \times 10^{-3}$. The thus defined region of interest (ROI) is the basis for the calculation of the $z$-profiles (dashed lines in Fig. 3). For each tube, the ROI had a dimension $(x, y, z)$ of $28 \times 1 \times 79$ voxels corresponding to $3.94 \mathrm{~mm} \times 1 \mathrm{~mm} \times 11.11 \mathrm{~mm}$ as the slice thickness in the sagittal slices was $1 \mathrm{~mm}$.

The principle expectation for the MRI signal intensity of the evolving sedimentation is summarized in [17, 27-29]. As a suspension contains equally, but randomly distributed particles, rather constant intensity of the liquid can be measured along $z$ (Fig. 4). As the particles continue settling over time, three regions are formed: suspension, clear liquid and sediment. These regions differ in particle concentration. Thus, the maximum signal intensity is measured in the liquid containing no particles. The lowest intensity is observed in the sediment layer. Between these two regions, the signal intensity varies due to various particle concentrations. After the particles settled completely, only particle poor and particle rich regions remain, which are reflected in the MRI $z$-profiles (Fig. 4).

The slight increase in intensity below the sediment layer in Fig. 4, i.e., outside the glass sample tube, is due to susceptibility artifacts due to LFP particles, which arise from the increasing concentration of these highly paramagnetic particles at the bottom. This issue will be discussed later in more detail.

The $z$-profiles, i.e., axial mean values, are a function of sedimentation time $t$ as the particle concentration 
Figure 3 Experimental setup: two samples were measured simultaneously in one sagittal slice. A PTFE sample holder was used to provide robust and aligned orientation of the tubes. High intensities correlate with low particle concentration and are attributed to the ${ }^{1} \mathrm{H}-\mathrm{MRI}$ signal of the liquid.

Table 2 MRI parameters of the 2D RARE MRI experiments

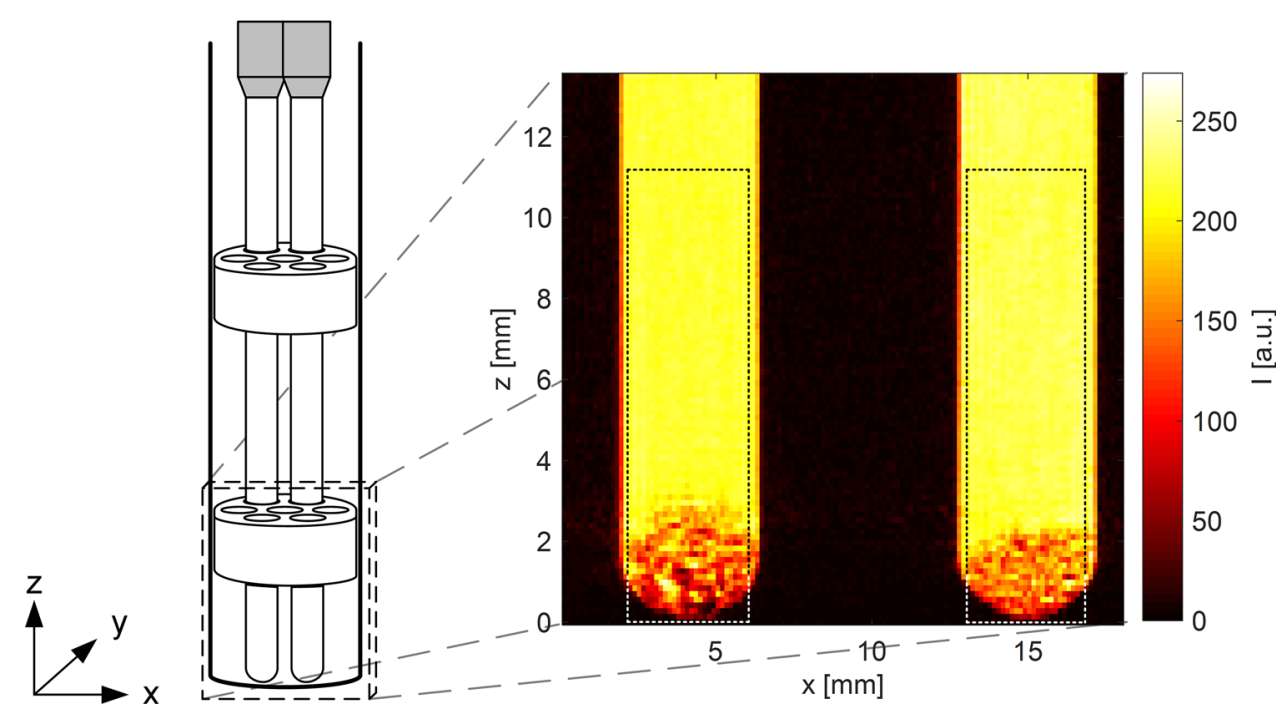

\begin{tabular}{ll}
\hline${ }^{1}$ H-RARE MRI parameter & Value \\
\hline Echo time & $10.16 \mathrm{~ms}$ \\
Repetition time & $2 \mathrm{~s}(\mathrm{CB}), 1 \mathrm{~s}(\mathrm{LFP})$ \\
Number of averages & $4(\mathrm{CB}), 2(\mathrm{LFP})$ \\
RARE factor & 4 \\
Acquisition time & $4: 16 \mathrm{~min}(\mathrm{CB}), 1: 04 \mathrm{~min}(\mathrm{LFP})$ \\
Field of view (FOV) & $18 \mathrm{~mm} \times 18 \mathrm{~mm}(128 \mathrm{px} \times 128 \mathrm{px})$ \\
Slice thickness of the sagittal slice & $1 \mathrm{~mm}$ \\
Resolution & $140.6 \mu \mathrm{m} \times 140.6 \mu \mathrm{m}$ \\
\hline
\end{tabular}

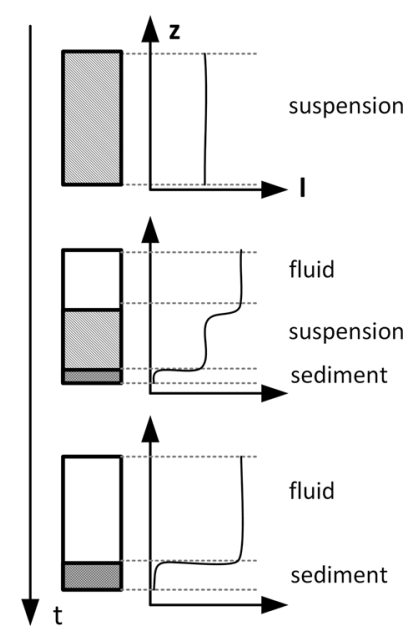

Figure 4 Scheme of NMR intensity profiles during sedimentation (modified from [27], left). In comparison, the measured $z$-profiles over $6 \mathrm{~h} 40 \mathrm{~min}$ are shown on the right. The experiment was performed on LFP particles with a volume fraction

changes with $t$ along $z$ (Fig. 5). Calculating horizontal mean values from unevenly distributed shapes of the settling front would result in a wrong interpretation

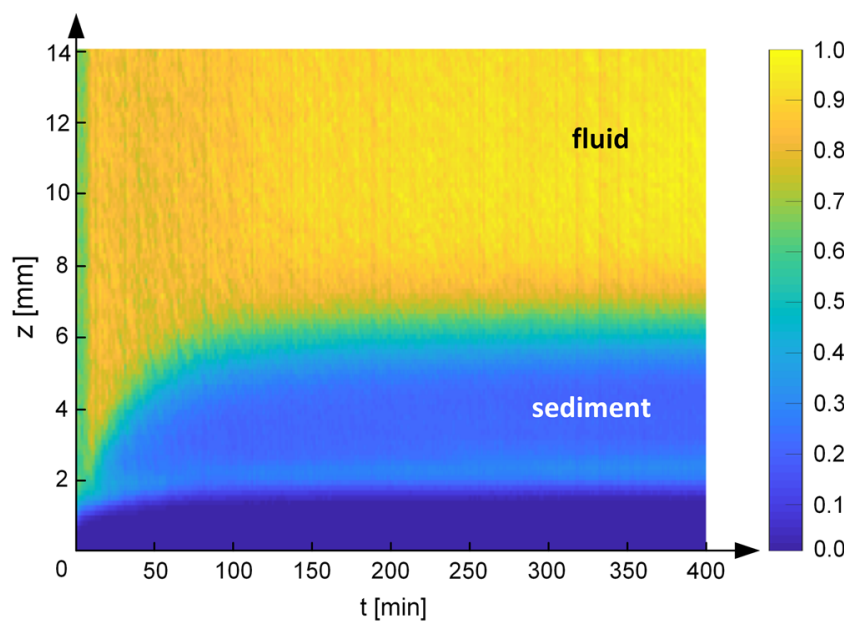

$\phi_{\mathrm{p}}=0.32 \mathrm{vol} \%$ in glycerin/water mixture $(80: 20 \mathrm{wt} \%)$ with a total volume of $1.55 \mathrm{ml}$. The evolution of the settling can be observed in the profiles. The bottom of the tube was at $z=1.13 \mathrm{~mm}$.

of the evolution of particle sedimentation along $z$. Thus, apart from the $z$-profiles, the $x$-profiles were also calculated to get an estimation about the shape of 

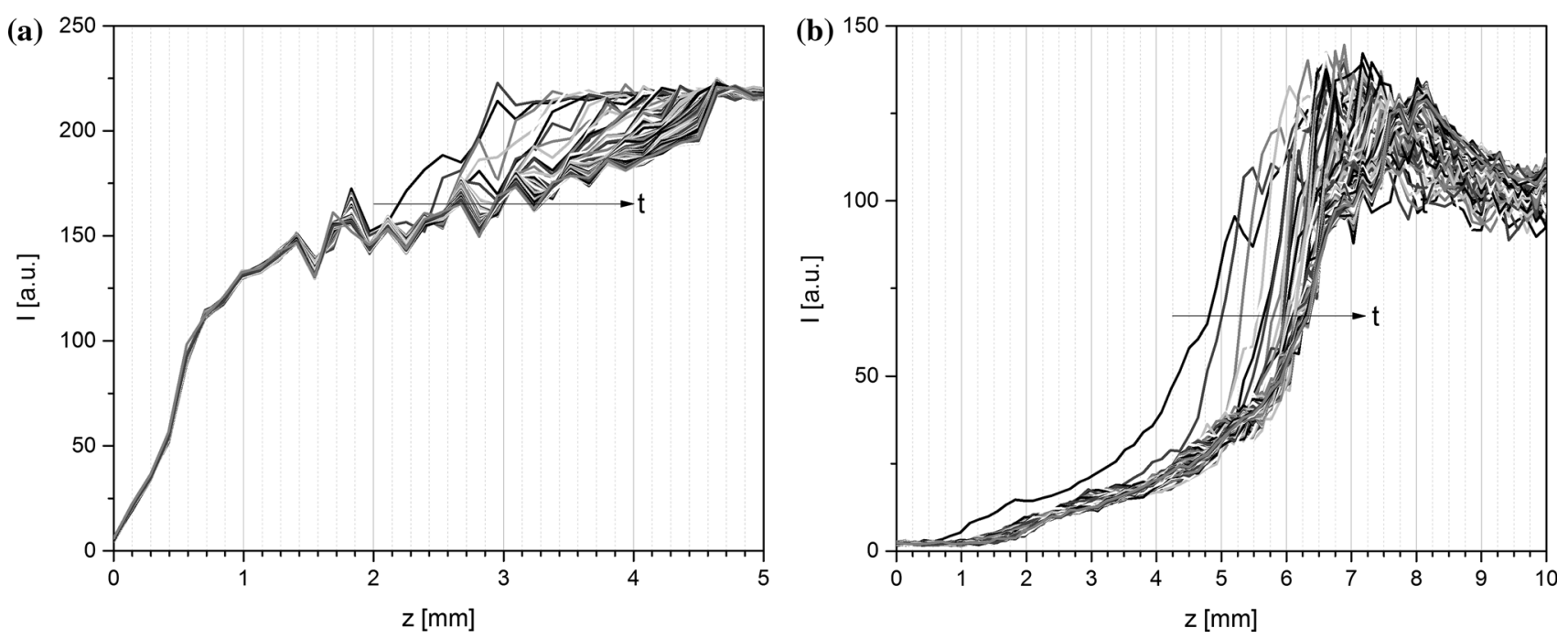

Figure 5 z-profiles as a function of sedimentation time $t$ of samples CB_1 (a) and LFP_2 (b). The higher the intensity, the lower the particle concentration. The total experiment time for sedimentation was $18 \mathrm{~h} 51 \mathrm{~min}$ for CB and $5 \mathrm{~h} 47 \mathrm{~min}$ for LFP.

the sediment's bed. Homogeneously distributed sedimentation over the cross section of the tube was observed; thus, wall effects can be neglected in the ROI of the $z$-profiles.

\section{Sedimentation balance}

A self-manufactured sedimentation balance was developed based on the standard DIN 66116-1:197311 and was used to measure the settling rate of the LFP particle suspensions and a commercial cathode slurry for LIB (Fig. 6).

A steel stage was placed over a digital balance. A glass container rests on top of the steel stage, decoupling the weight of the glass container from the balance. A perforated pan was mounted hanging from a frame, which is placed on top of the balance, using a steel wire. As a result, the weight on top of the pan was directly connected to the balance. In order to guarantee the retention of the settling LFP particles by the pan, a filter with a mesh size of $500 \mathrm{~nm}$ was attached on top. At first, the hanging pan was placed in the glass container, ensuring no contact. Subsequently, the suspension was poured into the glass container and the balance was tared. As the LFP particles settled on top of the pan, the weight was directed through the steel wire and frame to the balance. The balance was connected to a computer, which recorded the time-dependent weight increase using the software KERN Balance Connection 4. The weight was recorded each second for a total

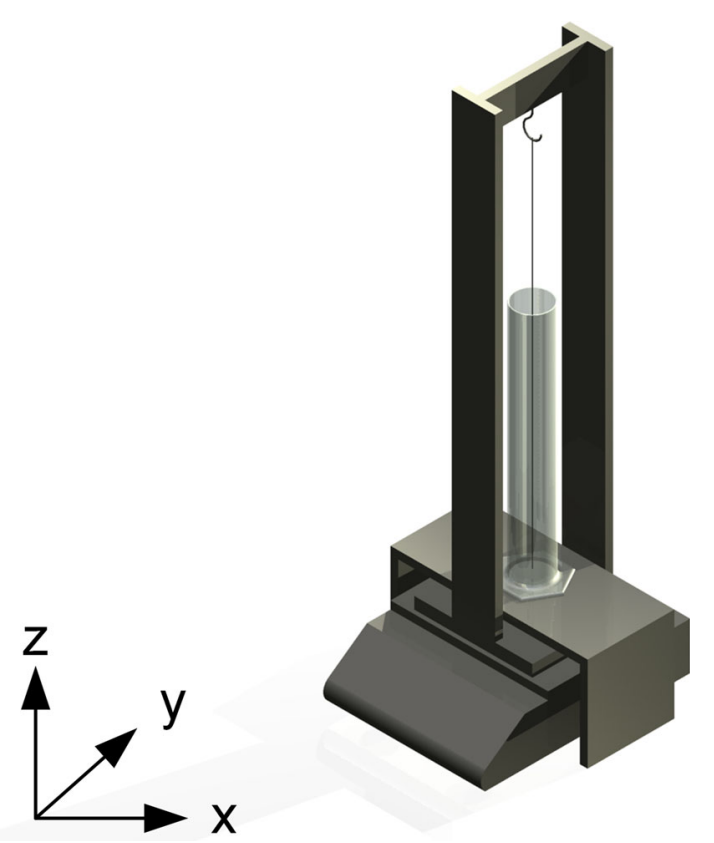

Figure 6 Schematic setup of the self-manufactured sedimentation balance based on standard DIN66116-1:1973-11.

experiment time of $4 \mathrm{~h} 10 \mathrm{~min}$ for each sample. Suspensions with LFP mass concentrations of $\phi_{\mathrm{m}}=10$, 30 and $50 \mathrm{wt} \%$ were dispersed in PDMS using a dissolver at various speeds between 1500 and $2000 \mathrm{rpm}$. 


\section{Results and discussion}

\section{MRI measurements}

The data set of $I(z, t)$ (Fig. 5) contains the complete information about sedimentation as the $x$-profiles (vertical mean values) showed no peak formation, but a uniform, evenly distributed intensity signal. Data processing can now be performed either along $z$ or $t$, in order to quantify the sedimentation process.

At first, the data are processed along $t$ to obtain $I(z=$ const, $t)$ : The intensity was computed as a function of sedimentation time for each of the 79 $z$-values measured for each sample. The form indicates an approximately linear dependence of $I$ on $t$. Similar behavior was observed for all samples of the same particle type, i.e., LFP or CB. For each $z$ value, $I$ is approximated by a linear function to extract the gradient $\frac{\Delta I(z, t)}{\Delta t}$ of the signal intensity over time (Fig. 7). The standard algorithm was applied to solve nonlinear curve-fitting problems in a least-square sense. The fitting parameters were calculated in the time range [0, 82] min for the sample CB_1 (Fig. 7). 0 min represents the first measurement, respectively, the first timestamp, which is approximately $2 \mathrm{~min}$ after sample preparation. The delay is caused by inserting the prepared sample into the probe and starting the experiment.

A mean value was calculated to define a threshold from the middle to the end of the data matrix, i.e.,

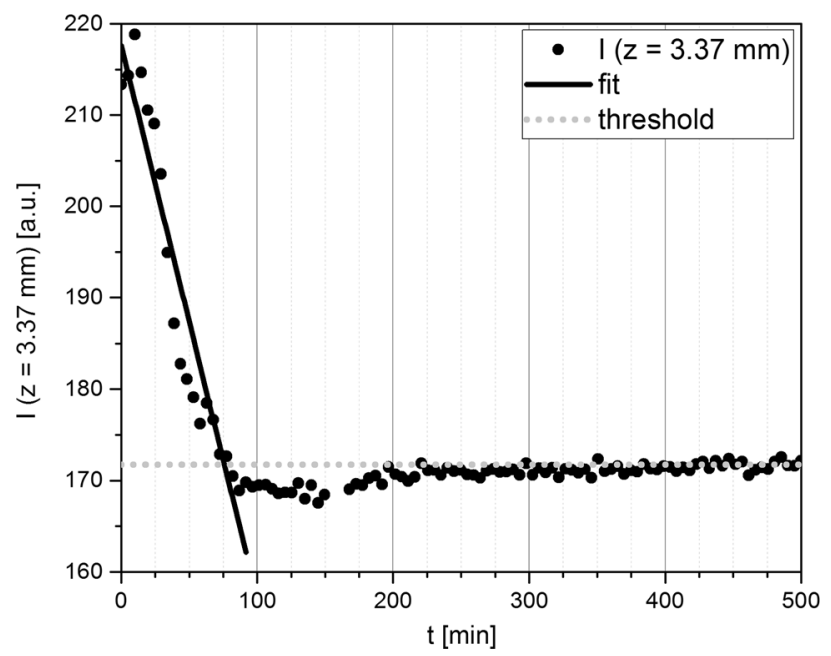

Figure 7 Signal intensity for $z=3.37 \mathrm{~mm}$ as a function of time $t$ for the $\mathrm{CB}$ particle sample $\mathrm{CB}_{-} 1$. The resulting profile is approximated by a linear function to extract the gradient of the signal intensity over $t$. large sedimentation times. The data matrix had 233 data points in total, respectively, 233 timestamps at a resolution of approximately $5 \mathrm{~min}$ for $\mathrm{CB}$ and $1.5 \mathrm{~min}$ for LFP. For better illustration, a zoom is depicted in Fig. 7. Starting from the first value, iterations compare the intensity values sequentially to the threshold. As soon as the value equals less than this threshold, the measured intensities at larger $t$-values are not considered in the linear fit. The threshold was adapted to each $z$-value automatically. As the ROI contained $79 z$-values, the same amount of iterations were computed for each of the eight samples. The change in intensity $\frac{\Delta I(z, t)}{\Delta t}$ as a function of $z$ gives insight into the reproducibility of sedimentation (Fig. 8). The gradients are in good agreement in each particle class, i.e., LFP or CB. Relatively time-constant concentration profiles (i.e., $\frac{\Delta I(z, t)}{\Delta t}=0$ ) are observed for top and bottom of the ROI. For the sedimentation of $\mathrm{CB}$, the most sensitive values of $\frac{\Delta I(z, t)}{\Delta t}$ are in the range $[2.5,4.0] \mathrm{mm}$; a second sensitive region was observed in the interval $[4.0,5.0] \mathrm{mm}$. This probably represents smaller intensity changes $\frac{\Delta I(z, t)}{\Delta t}$ due to slower sedimentation of small particles with lower settling velocities. Furthermore, the weighting error during sample preparation should be taken into account, especially at low particle mass fraction, i.e., $\phi_{\mathrm{p}}=0.5 \mathrm{wt} \%$.

In the case of LFP, the most sensitive region is observed for $z \in[4.0,7.0] \mathrm{mm}$; additionally, a signal enhancement at larger values of $z$ occurs between 7 and $8 \mathrm{~mm}$. An explanation of this pronounced difference within a material class can be found in the magnetic susceptibility $\chi_{\text {magn }}$. The most sensitive axis regarding $\chi_{\text {magn }}$ in MRI is the axis of the read gradient, which was chosen along $z$ in the experiments. As LFP is known to be paramagnetic, larger differences in $\chi_{\text {magn }}$ lead to spatially varying distortions of the main magnetic field of the NMR instrument with the consequence that NMR intensity shifts to other volume elements, leading to larger signal intensities in neighboring regions $z>7 \mathrm{~mm}$ (Fig. 8). Please note that some $10 \mathrm{ppm}$ is sufficient for this type of artifacts in MRI [30-35]. Similar behavior of $\frac{\Delta I(z, t)}{\Delta t}$ was observed. Negative values represent declining signal intensities. Therefore, particles settle in this region.

The next step to process the experimental data (Fig. 5) is to monitor the $z$-profiles and to deduce the sedimentation velocity by analyzing the intensity 

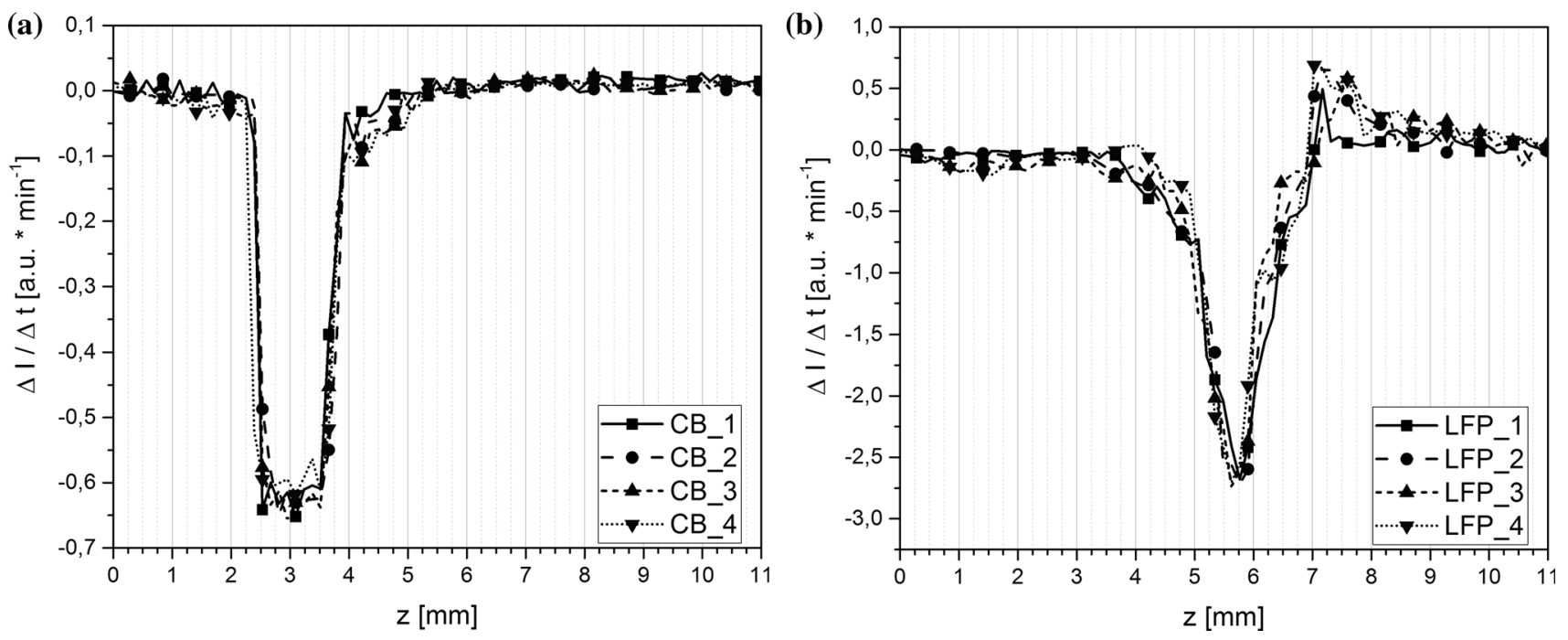

Figure 8 The gradient $\frac{\Delta I(z, t)}{\Delta t}$ reflects the sensitive regions and gives an estimate of the reproducibility of the MRI measurements. The most sensitive region for $\mathrm{CB}(\mathbf{a})$ is found in the interval $[2.5,4.0] \mathrm{mm}$, for LFP (b) in the region $[4.0,7.0] \mathrm{mm}$. The lines are guides to the eye.

isolines $I(z, t)=$ const. The basis for the calculation of the sedimentation velocity is $z(t, I=$ const) (Fig. 9). The isolines were only computed at intensity steps of $\Delta I=10$ a.u. for both samples. In detail, the intervals were for $\mathrm{CB}$ at $I \in[150,210]$ a.u. and at $I \in[20,80]$ a.u. for LFP.

Since the measured data are discrete with limited signal-to-noise ratio and due to the required time discretization of the measurement, each signal intensity profile was interpolated and therefore smoothed with a polynomial of the order $n=9$ for

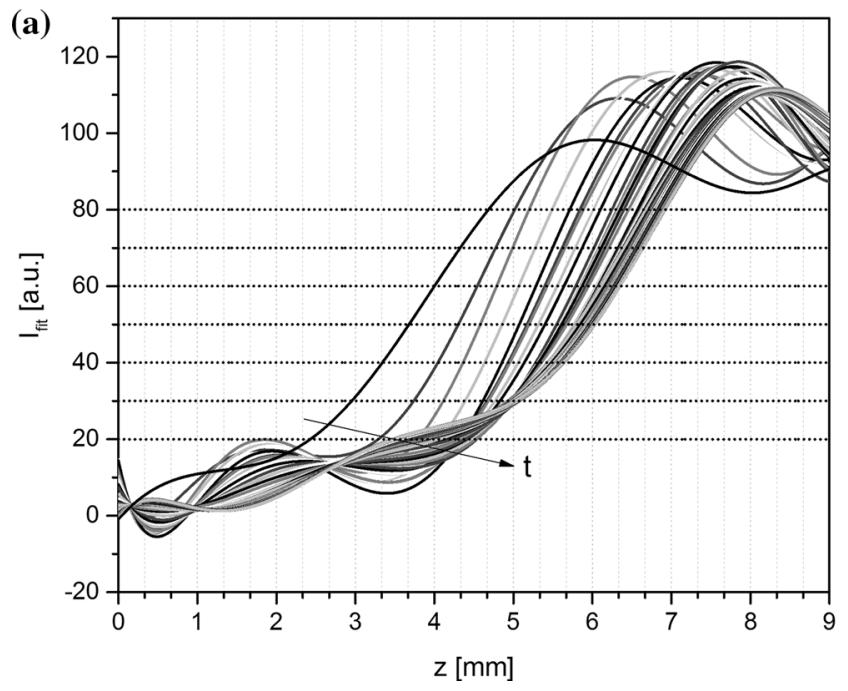

Figure 9 a Signal intensity profiles $I_{\text {fit }}(z, t)$ as derived from polynomial fits to experimental data $I_{\exp }(z, t)$ of the sample LFP_3. Each profile represents a timestamp. The settling front was
CB and $n=7$ for LFP. The high polynomial degrees were chosen to maintain the overall shape of the profile, i.e., to keep the signatures of the experimental data observed (Fig. 9), while removing artifacts and statistical noise. The settling velocity was calculated by considering the determined time difference between each profile from the timestamp and generating the required distance in $z$ direction by subtraction of the respective intersections. The quotient of $\Delta z / \Delta t$ provides the desired item and declines with t.

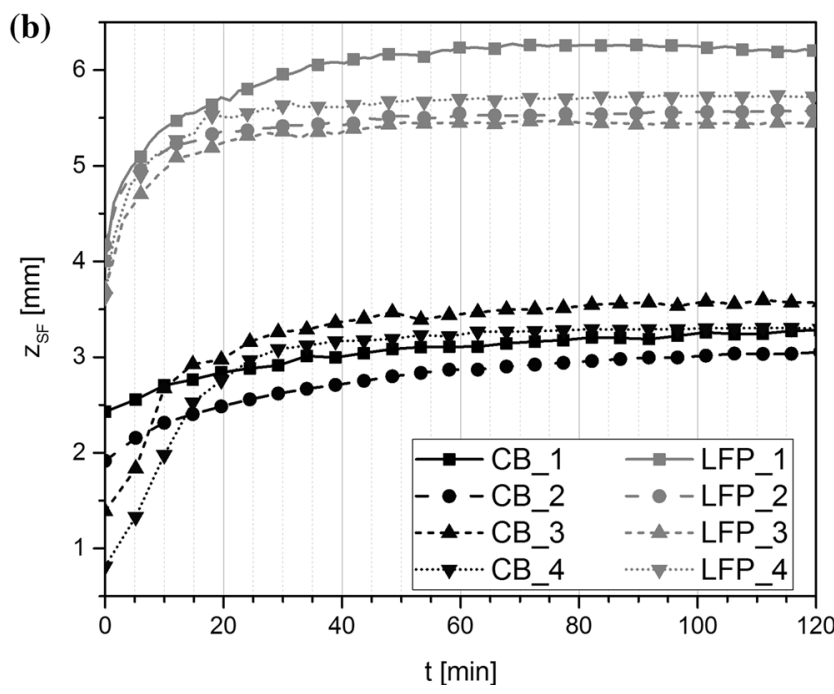

observed from the intersections of the isolines with the profiles. b The progression of the settling front along $z$ with $t$ for all measured samples. 
Sedimentation is a dynamic process, and the velocity is expected to be a function of sedimentation time. Therefore, $v_{\text {mean }}=\Delta z / \Delta t$ was modeled by an exponentially decaying function. The fit describes the measured data well within the experimental error. Large particles with high velocities settle first and form the initial growth of the sediment. Furthermore, they entrain smaller particles and accelerate them or induce agglomeration. The resulting effect is a primary, rather large velocity, which declines as smaller and less agglomerated particles remain in the suspension (Fig. 10).

Further experiments with mechanically homogeneously dispersed particles were performed, but showed another aspect of the investigated material. As instruments exploring Fraunhofer diffraction use an ultrasonic wet dispersing unit and is mainly sensitive to agglomerates in the order of several $\mu \mathrm{m}$, the nanoscale of single particle is not considered. CB and LFP particles are specifically manufactured with average diameters $<1 \mu \mathrm{m}$. Nanomaterials play a key role in the improvement of performance and energy density of LIB, since the overall thermal and electric DC conductivity increase with reciprocal particle size [36]. When CB and LFP are dispersed into PDMS at for instance the mentioned viscosity, i.e., $20 \mathrm{~mm}^{2} \mathrm{~s}^{-1}$, by using a dissolver for a certain duration, e.g., $>15 \mathrm{~min}$, and various speeds in the range 1500-2000 rpm, the majority of the agglomerates are separated. This results in a rather homogeneous and statistical distribution of the nanoscale particles.
Sedimentation at low and even at higher particle suspension fractions was not observable for the dissolved CB and LFP. Experiments were performed with $1 \mathrm{ml}$ of PDMS and $0.5 \mathrm{wt} \%$ dispersed CB as well as LFP. Intensity profiles were measured for more than $65 \mathrm{~h}$, but no sedimentation was observable by MRI. It seems natural that the counterforce of the liquid restrains the nanoparticles on a large scale. Furthermore, particle diffusion needs to be taken into account on this length scale of particle diameters. Increasing the particle fraction in the suspension only leads to high viscosities and therefore to even lower theoretical sedimentation rates. Hence, reasonable limits can be outlined for the presented spin-echobased MRI method: certain viscosities need to be maintained to match MRI measurement time with the sedimentation time scale. The MRI timescale is thereby given by mainly the repetition time, the number of averages and the number of phase steps which could be reduced to $5-10$, leading to a measurement time reduction by a factor of two at least. Furthermore, minimum as well as maximum particle suspension fraction and the PSD need to be considered. Increasing LFP particle mass fractions in MRI experiments would also increase the effect of signal intensity distortion. Hence, a valuable compromise needs to be achieved.
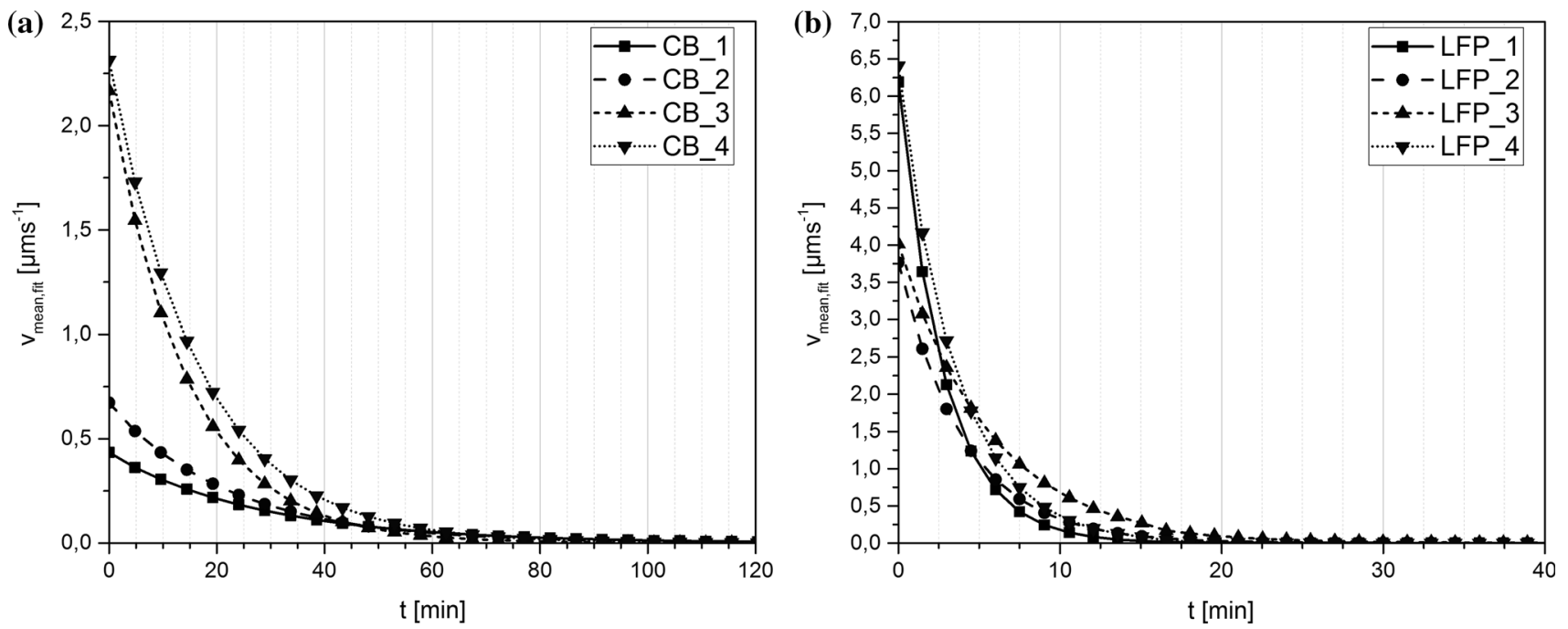

Figure 10 Settling velocities of the model with exponentially decaying function for CB (a) and LFP (b). The deviation for the velocities within the two material classes is a result of small variations of the particle mass fraction $\phi_{\mathrm{p}}$ and of particle size distribution. 


\section{Sedimentation balance}

Supplementing the MRI measurements, suspensions based on PDMS with LFP mass fractions of $\phi_{\mathrm{m}}=10$, 30 and $50 \mathrm{wt} \%$ were studied. The measured mass concentration $w_{i}$ represents the settled mass fraction in relation to the total solid mass in the suspension, i.e., $w_{i}=\frac{m_{i}}{m_{\Sigma, \text { solid }}}$. The LFP slurries of different concentrations as well as a commercial cathode paste show changes of $w_{i}$ over time (Fig. 11). In all cases the slope of $w_{i}(t)$ decreases with settling time. Larger particles or agglomerates sediment rapidly, resulting in a prompt increase in the settled mass concentration at short measuring times. Homogeneously dispersed particles and small agglomerates sediment more slowly. The sedimentation behavior, i.e., the decay of the measured mass concentration over time, described by the sedimentation balance is in good agreement with the settling behavior characterized by MRI (Fig. 10), where the settling velocities exponentially decline over time. The slope of the curves at short measuring times $(<20 \mathrm{~min})$ decreases as the solid content in the suspension increases, suggesting higher settling velocities for diluted LFP suspensions at short measuring times. Furthermore, the total amount of settled LFP particles decreases.

At a particle concentration of $10 \mathrm{wt} \%$, the viscosity of the suspension is low enough and particles can

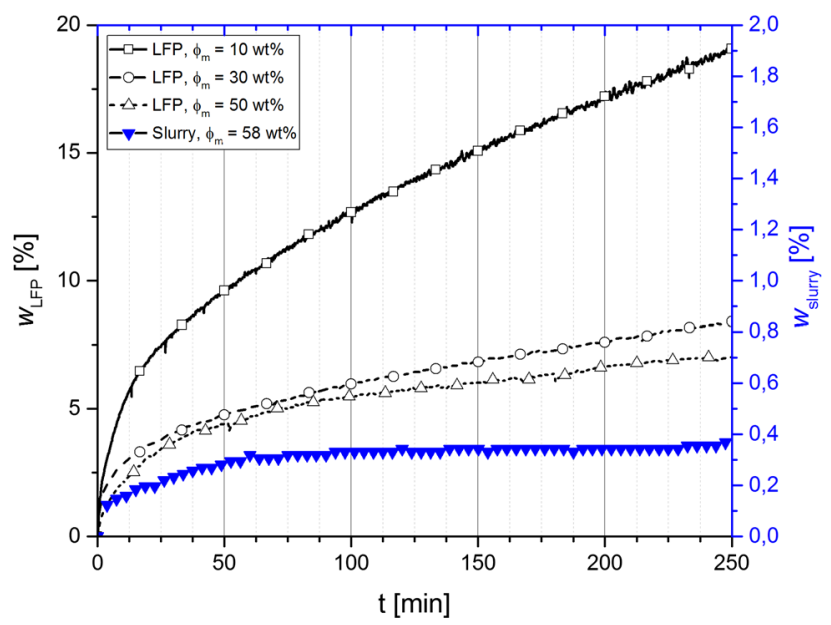

Figure 11 Mass concentration profiles over time $t$ for LFP suspensions with particle mass fractions $\phi_{\mathrm{m}}=10,30$ and $50 \mathrm{wt} \%$ measured with the sedimentation balance. The profile of the aqueous cathode slurry including the organic binder material CMC and a total suspension mass fraction $\phi_{\mathrm{m}}=58 \mathrm{wt} \%$ show a weak increase in measured mass due to the higher low shear viscosity of the slurry compared to the pure suspensions (Fig. 2). settle on the pan. Increasing the solid fraction results in a strong increase in viscosity [37-39]. Thus, highly concentrated suspensions show overall lower sedimentation velocities. It should be noted that not all particles settle on top of the pan. In order to avoid contact between the pan and the glass container, the diameter of the pan and the attached filter is slightly smaller than that of the container. Hence, the pan covers $70 \%$ of the total cross-sectional area. Although the LFP particles cannot be measured in their entirety, the differences in measured mass changes are still representative and render a reasonable relative measure of sedimentation behavior.

Regarding the industrial application of the type of suspensions presented in this work, the settling behavior of highly concentrated electrode slurries, including various additives such as binders, dispersants and rheology control agents, is of great interest. Consequently, a water-based cathode slurry with LFP as active material was investigated (Fig. 11). The sedimentation of the aqueous cathode slurry, i.e., the decay of measured solid mass concentration over time, is similar to that of LFP suspensions. The total amount of settled particles, i.e., the increase in mass concentration $w_{i}$, however, is more than one order of magnitude lower compared to the results obtained for LFP suspensions. The electrode slurry consists of active material, carbon black as conductive agent and $\mathrm{CMC}$, which acts as a dispersing agent for LFP and $\mathrm{CB}$ and also as a thickener increasing the viscosity of the continuous phase [40-43]. Both mechanisms stabilize the slurry against sedimentation resulting in the much lower weight gain compared to the pure slurries shown in Fig. 11.

\section{Conclusion}

Spin-echo-based MRI experiments were performed to characterize LFP and CB particles in opaque suspensions used for LIB electrodes at a particle mass fraction of $\phi_{\mathrm{p}}=0.5 \mathrm{wt} \%$. The presented method of processing the intensity profiles from the MR images yields good reproducibility throughout the samples. The sedimentation velocity for the two particle classes was derived from the measured evolution of a settling front and modeled by an exponentially decaying function. Detailed insights into the sedimentation of opaque suspensions are provided, since the ${ }^{1} \mathrm{H}$ images uncover the spatial distribution of the 
particles during sedimentation. Supplementing the MRI experiments, the sedimentation of highly concentrated LFP suspensions was investigated using a self-manufactured sedimentation balance. LFP mass fractions of $\phi_{\mathrm{m}}=10,30$ and $50 \mathrm{wt} \%$ were measured. The time-dependent decay of the sedimentation velocity measured by MRI is in good agreement with the increase in the settled mass concentration measured by the gravimetrical method. Inter-particle interactions at higher solid fractions result in a strong increase in the sedimentation viscosity.

Moreover, the sedimentation behavior of an aqueous cathode slurry with LFP as active material and $\mathrm{CB}$ as conductive additive was investigated for a realistic and industrially applicable total mass fraction including all components of $\phi_{\mathrm{m}}=58 \mathrm{wt} \%$. Although the sedimentation behavior is similar to the one measured for LFP suspensions, the total amount of settled particles is more than one order of magnitude lower. This is attributed to the added polymer serving as a dispersing agent and as a thickener for the continuous phase, thus slowing down sedimentation significantly. Hence, the presented measuring methods provide a reliable combination in order to characterize the sedimentation of opaque suspensions of LFP and CB particles with different particle mass fractions. The focus of this paper was on the development of the experiments and the methods. Future work will address the comparison between the experimental data and the theoretical sedimentation behavior, i.e., force balance for colloidal particles.

\section{Acknowledgements}

The authors would like to thank the German Research Foundation (DFG) for financial support of the instrumental facility Pro ${ }^{2} \mathrm{NMR}$. Furthermore, the authors thank the DFG for funding and the cooperative support within GRK 2218 SiMET-Simulation of mechano-electro-thermal processes in lithium-ionbatteries, project number: 281041241). R. Gordon gratefully acknowledges financial support by the 100 prozent erneuerbar Stiftung.

\section{Compliance with ethical standards}

Conflict of interest The authors declare no conflicts of interest.

\section{References}

[1] Chikkannanavar SB, Bernardi DM, Liu L (2014) A review of blended cathode materials for use in Li-ion batteries. J Power Sour 248:91. https://doi.org/10.1016/j.jpowsour.20 13.09.052

[2] Wu H, Cui Y (2012) Designing nanostructured Si anodes for high energy lithium ion batteries. Nano Today 7:414. http s://doi.org/10.1016/j.nantod.2012.08.004

[3] Berla LA, Lee SW, Ryu I, Cui Y, Nix WD (2014) Robustness of amorphous silicon during the initial lithiation/delithiation cycle. J Power Sourc 258:253. https://doi.org/10.1016/j.jpo wsour.2014.02.032

[4] Zeng Z, Liu N, Zeng Q, Ding Y, Qu S, Cui Y, Mao WL (2013) Elastic moduli of polycrystalline Li15Si4 produced in lithium ion batteries. J Power Sourc 242:732. https://doi.org/ 10.1016/j.jpowsour.2013.05.121

[5] Bogart TD, Chockla AM, Korgel BA (2013) High capacity lithium ion battery anodes of silicon and germanium. Curr Opin Chem Eng 2:286. https://doi.org/10.1016/j.coche.2013. 07.001

[6] Zuo X, Zhu J, Müller-Buschbaum P, Cheng Y-J (2017) Silicon based lithium-ion battery anodes: a chronicle perspective review. Nano Energy 31:113. https://doi.org/10.1016/j. nanoen.2016.11.013

[7] Chen T, Wu J, Zhang Q, Su X (2017) Recent advancement of $\mathrm{SiO}_{x}$ based anodes for lithium-ion batteries. J Power Sour 363:126. https://doi.org/10.1016/j.jpowsour.2017.07.073

[8] Goriparti S, Miele E, De Angelis F, Di Fabrizio E, Proietti Zaccaria R, Capiglia C (2014) Review on recent progress of nanostructured anode materials for Li-ion batteries. J Power Sour 257:421. https://doi.org/10.1016/j.jpowsour.2013.11. 103

[9] Xu J, Dou S, Liu H, Dai L (2013) Cathode materials for next generation lithium ion batteries. Nano Energy 2:439. http s://doi.org/10.1016/j.nanoen.2013.05.013

[10] Scrosati B, Garche J (2010) Lithium batteries: status, prospects and future. J Power Sour 195:2419. https://doi.org/10. 1016/j.jpowsour.2009.11.048

[11] Prosini PP, Lisi M, Zane D, Pasquali M (2002) Determination of the chemical diffusion coefficient of lithium in $\mathrm{LiFePO}_{4}$. Solid State Ion 148:45. https://doi.org/10.1016/S 0167-2738(02)00134-0

[12] Andersson AS, Kalska B, Häggström L, Thomas JO (2000) Lithium extraction/insertion in $\mathrm{LiFePO}_{4}$ : an X-ray diffraction and Mössbauer spectroscopy study. Solid State Ion 130:41. https://doi.org/10.1016/S0167-2738(00)00311-8

[13] Kenney B, Darcovich K, MacNeil DD, Davidson IJ (2012) Modelling the impact of variations in electrode 
manufacturing on lithium-ion battery modules. J Power Sour 213:391. https://doi.org/10.1016/j.jpowsour.2012.03.065

[14] Li J, Armstrong BL, Daniel C, Kiggans J, Wood DL (2013) Optimization of multicomponent aqueous suspensions of lithium iron phosphate $\left(\mathrm{LiFePO}_{4}\right)$ nanoparticles and carbon black for lithium-ion battery cathodes. J Colloid Interface Sci 405:118. https://doi.org/10.1016/j.jcis.2013.05.030

[15] Zăbavă B, Ungureanu N, Vlădut V, Dincă M, Voicu G, Ionescu M (2016) Experimental study of the sedimentation of solid particles in wastewater. In: Annals of the University of Craiova-Agriculture, Montanology, Cadastre Series, vol 46, p 611

[16] Lu W-M, Tung K-L, Pan C-H, Hwang K-J (1998) The effect of particle sedimentation on gravity filtration. Sep Sci Technol 33:1723. https://doi.org/10.1080/ 01496399808545902

[17] Turney MA, Cheung MK, Powell RL, McCarthy MJ (1995) Hindered settling of rod-like particles measured with magnetic resonance imaging. AIChE J 41:251. https://doi.org/10. 1002/aic.690410207

[18] Morozov EV, Shabanova OV, Falaleev OV (2013) MRI comparative study of container geometry impact on the PMMA spheres sedimentation. Appl Magn Reson 44:619. h ttps://doi.org/10.1007/s00723-012-0432-8

[19] Acosta-Cabronero J, Hall LD (2009) Measurements by MRI of the settling and packing of solid particles from aqueous suspensions. AIChE J 55:1426. https://doi.org/10.1002/aic. 11754

[20] Bürger R, Wendland WL, Concha F (2000) Model equations for gravitational sedimentation-consolidation processes. ZAMM J Appl Math Mech 80:79. https://doi.org/10.1002/ (SICI)1521-4001(200002)80:2\%3c79:AID-ZAMM79\%3e3. 0.CO;2-Y

[21] Lehtimäki M, Keskinen J, Janka K (1990) Sedimentation method in calibrating optical particle counters. Aerosol Sci Technol 12:711. https://doi.org/10.1080/ 02786829008959386

[22] Azema N (2006) Sedimentation behaviour study by three optical methods-granulometric and electrophoresis measurements, dispersion optical analyser. Powder Technol 165:133. https://doi.org/10.1016/j.powtec.2005.10.015

[23] Lerche D (2002) Dispersion stability and particle characterization by sedimentation kinetics in a centrifugal field. J Dispers Sci Technol 23:699. https://doi.org/10.1081/DIS120015373

[24] Detloff T, Sobisch T, Lerche D (2007) Particle size distribution by space or time dependent extinction profiles obtained by analytical centrifugation (concentrated systems). Powder Technol 174:50. https://doi.org/10.1016/j.powtec.2 006.10.021
[25] Rwei S-P, Ku F-H, Cheng K-C (2002) Dispersion of carbon black in a continuous phase: electrical, rheological, and morphological studies. Colloid Polym Sci 280:1110. http s://oi.org/10.1007/s00396-002-0718-8

[26] Kawaguchi M, Okuno M, Kato T (2001) Rheological properties of carbon black suspensions in a silicone oil. Langmuir 17:6041. https://doi.org/10.1021/la010560r

[27] Bobroff S, Phillips RJ (1998) Nuclear magnetic resonance imaging investigation of sedimentation of concentrated suspensions in non-Newtonian fluids. J Rheol 42:1419. h ttps://doi.org/10.1122/1.550895

[28] Cheung MK, Powell RL, McCarthy MJ (1996) Sedimentation of noncolloidal bidisperse suspensions. AIChE J 42:271. https://doi.org/10.1002/aic.690420125

[29] Turney MA, Cheung MK, McCarthy MJ, Powell RL (1995) Magnetic resonance imaging study of sedimenting suspensions of noncolloidal spheres. Phys Fluids 7:904. https://doi. org/10.1063/1.868566

[30] Tucker MC, Doeff MM, Richardson TJ, Fiñones R, Reimer JA, Cairns EJ (2002) 7Li and $31 \mathrm{P}$ magic angle spinning nuclear magnetic resonance of $\mathrm{LiFePO}_{4}$-type materials. Electrochem Solid State Lett 5:A95. https://doi.org/10.1149/ 1.1464505

[31] Grey CP, Dupré N (2004) NMR studies of cathode materials for lithium-ion rechargeable batteries. Chem Rev 104:4493. https://doi.org/10.1021/cr020734p

[32] Tucker MC, Doeff MM, Richardson TJ, Fiñones R, Cairns EJ, Reimer JA (2002) Hyperfine fields at the Li site in $\mathrm{LiFePO}_{4}$-type olivine materials for lithium rechargeable batteries: a 7Li MAS NMR and SQUID study. J Am Chem Soc 124:3832. https://doi.org/10.1021/ja017838m

[33] Wilcke SL, Lee Y-J, Cairns EJ, Reimer JA (2007) Covalency measurements via NMR in lithium metal phosphates. Appl Magn Reson 32:547. https://doi.org/10.1007/s00723-007-0 032-1

[34] Buzlukov A, Gerbaud G, Bourbon C, Hediger S, De Paëpe G, Patoux S, Bardet M (2013) Application of 7Li NMR to characterize the evolution of intercalated and non-intercalated lithium in $\mathrm{LiFePO}_{4}$-based materials for Li-ion batteries. J Solid State Electr 17:1421. https://doi.org/10.1007/s10008013-2011-9

[35] Dupré N, Oliveri J, Degryse J, Martin J-F, Guyomard D (2008) Characterization of the surface of positive electrodes for Li-ion batteries using 7Li MAS NMR. Ionics 14:203. h ttps://doi.org/10.1007/s11581-007-0189-5

[36] Satyavani TVSL, Ramya Kiran B, Rajesh Kumar V, Srinivas Kumar A, Naidu SV (2016) Effect of particle size on dc conductivity, activation energy and diffusion coefficient of lithium iron phosphate in Li-ion cells. Eng Sci Technol Int J 19:40. https://doi.org/10.1016/j.jestch.2015.05.011 
[37] Krieger IM (1959) A mechanism for non-newtonian flow in suspension of rigid spheres. Trans Soc Rheol 3:137-152. h ttps://doi.org/10.1122/1.548848

[38] Quemada D (1977) Rheology of concentrated disperse systems and minimum energy dissipation principle. Rheol Acta 16:82. https://doi.org/10.1007/BF01516932

[39] Meeker SP, Poon WCK, Pusey PN (1997) Concentration dependence of the low-shear viscosity of suspensions of hard-sphere colloids. Phys Rev E 55:5718. https://doi.org/ 10.1103/PhysRevE.55.5718

[40] Tsai F-Y, Jhang J-H, Hsieh H-W, Li C-C (2016) Dispersion, agglomeration, and gelation of $\mathrm{LiFePO}_{4}$ in water-based slurry. J Power Sour 310:47. https://doi.org/10.1016/j.jpow sour.2016.02.003
[41] Lee J-H, Kim J-S, Kim YC, Zang DS, Paik U (2008) Dispersion properties of aqueous-based $\mathrm{LiFePO}_{4}$ pastes and their electrochemical performance for lithium batteries. Ultramicroscopy 108:1256. https://doi.org/10.1016/j.ultrami c.2008.04.027

[42] Li C-C, Peng X-W, Lee J-T, Wang F-M (2010) Using poly(4-styrene sulfonic acid) to improve the dispersion homogeneity of aqueous-processed $\mathrm{LiFePO}_{4}$ cathodes. J Electrochem Soc 157:A517. https://doi.org/10.1149/1. 3308595

[43] Guerfi A, Kaneko M, Petitclerc M, Mori M, Zaghib K (2007) $\mathrm{LiFePO}_{4}$ water-soluble binder electrode for Li-ion batteries. J Power Sour 163:1047. https://doi.org/10.1016/j.jpowsour. 2006.09.067 
Karlsruher Institut für Technologie

\section{Repository KITopen}

Dies ist ein Postprint/begutachtetes Manuskript.

Empfohlene Zitierung:

Balbierer, R.; Gordon, R.; Schuhmann, S.; Willenbacher, N.; Nirschl, H.; Guthausen, G. Sedimentation of lithium-iron-phosphate and carbon black particles in opaque suspensions used for lithium-ion-battery electrodes. 2019. Journal of materials science, 54 . doi: $\underline{10.5445 / / R / 1000097070}$

Zitierung der Originalveröffentlichung:

Balbierer, R.; Gordon, R.; Schuhmann, S.; Willenbacher, N.; Nirschl, H.; Guthausen, G. Sedimentation of lithium-iron-phosphate and carbon black particles in opaque suspensions used for lithium-ion-battery electrodes.

2019. Journal of materials science, 54 (7), 5682-5694.

doi: $10.1007 / \mathrm{s} 10853-018-03253-2$ 